\title{
DIAGRAMS FOR OBSERVATIONAL TESTING OF EVOLUTION OF PLANETARY NEBULA NUCLEI
}

\author{
R. TYLENDA \\ Copernicus Astronomical Center, Laboratory of Astrophysics \\ ul. Chopina 12/18, 87-100 Toruń, Poland
}

\begin{abstract}
Confrontation of theoretical models with observations is the main way of progress of our understanding of the nature of astrophysical objects. This paper discusses different diagrams used for observational testing of theoretical evolutionary models of planetary nebula nuclei (PN$\mathrm{Ni}$ ). Particular attention is paid in indicating and discussing sources of errors and uncertainties which may result in wrong conclusions drawn from the diagrams. Finally, results of recent studies comparing the theory and observations for the Galactic PNNi are critically reviewed.
\end{abstract}

\section{H-R diagram}

This is the most classic and most widely applied diagram for observational testing of the theory of stellar evolution and of the PNN evolution, in particular. The most often used version of this diagram plots the PNN luminosity, $L_{\star}$, versus the PNN effective temperature, $T_{\star}$. Thus the main advantage of the $\mathrm{H}-\mathrm{R}$ diagram is that it displays fundamental parameters of a PNN, parameters whose physical meaning and interpretation are obvious and clear. Moreover, these parameters can be reliably determined from theoretical model calculations.

Unfortunately, from an observational point of view the problem is subject to substantial uncertainties. In the case of a typical PNN the bulk of energy is emitted in the EUV region which cannot be directly observed. As a result, determination of $T_{\star}$ of a PNN is usually difficult and uncertain. This subject is discussed in detail in another review (Preite-Martinez in this volume). As shown by Schönberner \& Tylenda (1990) errors in $T_{\star}$ can be important, especially if the observational material is poor or if one uses not entirely correct assumptions (e.g. that all nebulae are optically thick). As a result the obtained positions in the H-R diagram may be very different from the true ones leading to incorrect conclusions.

The errors in $T_{\star}$ have also important consequences on the PNN luminosity. This is because $L_{\star}$ is often calculated from an observed PNN magnitude and a bolometric correction; the latter being a strong function of the adopted $T_{\star}$.

Another way of calculating $L_{\star}$ is to use nebular $H \beta$ or radio fluxes. These quantities can be measured with a relatively high precision which is not often the case for the PNN magnitudes (e.g. problems with nebular contamination). Another advantage is that apart from the coolest $\mathrm{PNNi}$ the relation between the $H \beta$ (or radio) flux and the PNN luminosity is only weakly dependent on $T_{\star}$. However, this method gives reliable results only if the nebula completely absorbs the ionizing radiation. Otherwise one gets only a lower limit to $L_{\star}$.

Observational verification of a question whether a particular planetary nebula (PN) completely absorbs the PNN ionizing flux or not, is not a straightforward problem. Strong lines of low excitation ([NII], [OII]) are sometimes taken as a criterion for optically thick PNe. However, for a non-spherical PN - which is often the case - strong low-excitation lines may indicate that the PN is indeed optically 
thick but only in some directions. In some cases - especially for old, low luminosity objects - when the so-called ionizing parameter is low, the nebular gas is mildly ionized. Then even in innermost $\mathrm{PN}$ regions there is an important proportion of neutral or singly ionized species and the strength of low-excitation lines is a measure of the ionizing parameter rather than of the optical thickness.

Some authors interpret an observed increase of the ionized PN mass, $M_{\text {ion }}$, with the nebular radius, $R_{P N}$, as an argument that the $\mathrm{PNe}$ are opaque (e.g. Pottasch \& Acker 1989; Zijlstra \& Pottasch 1989). However, this correlation has to be interpreted with a great caution since $M_{i o n}$ and $R_{P N}$ are not independent. This fact in combination with observational selection effects and uncertainties in observed quantities can produce an apparent trend of increasing $M_{i o n}$ with $R_{P N}$ no matter whether the PNe are optically thick or thin (Stasinska et al. 1991a; Zijlstra 1990; Stasińska \&. Tylenda in this volume).

As usually in the case of stellar luminosities a crucial problem lies in the PN distances. Another review is devoted to this subject (Terzian in this volume). The present situation in this field is far from being satisfactory and is a source of substantial errors and uncertainties. Hence importance of investigations of PN samples with fairly accurate distances, i.e. Galactic bulge, Magellanic Clouds.

Another source of uncertainties is the interstellar extinction. A common opinion seems to be that in the case of $\mathrm{PNe}$ the extinction can be determined quite accurately, e.g. from an observed Balmer decrement. Then the observations can be corrected without particular problems, at least in the optical. However, it has recently been found that the extinction from Balmer decrement, $C_{o p t}$, is often larger than that from the radio/ $H \beta$ flux ratio, $C_{\text {rad }}$ (Stasinska et al. 1991a; Cahn et al. 1992; Tylenda et al. 1992). A thorough discussion of the problem in Stasinska et al. (1992) reveals that there is a systematic trend between the two values with an average relation $C_{o p t} \simeq 1.17 C_{\text {rad }}$. The origin of this effect is not clear. Nevertheless, this shows that depending on the method for determining extinction one can obtain a systematic shift in luminosity by factor 2 for heavily reddened PNe.

In conclusion, placing an observed PNN on the H-R diagram is not a straightforward or easy problem. There are numerous sources of errors while determining $T_{\star}$ and $L_{\star}$. If this is combined with the fact that theoretical tracks are usually close one to another in the $\log L_{\star}-\log T_{\star}$ plane (cf. dashed curves in Fig.1a) it is clear that an estimation of PNN masses from observed positions in the H-R diagram can be subject to large uncertainties. In addition, some objects may appear in a region where no theoretical track passes through. Therefore, conclusions have to be drawn with a great caution while interpreting an observed PNN H-R diagram

\section{2. $M_{v}-t_{e v}$ diagram}

Theoretical models of the PNN evolution predict that the evolutionary time scale strongly decreases with the PNN mass (Schönberner 1979, 1983; Wood \& Faulkner 1986). This fact offers an important possibility for observational testing of PNN models. Schönberner (1981) was first to explore this possibility using a plot of absolute visual PNN magnitude, $M_{v}$, versus PN radius, $R_{P N}$, originally proposed by Abell (1966). 
Principal advantages and disadvantages of the $M_{v}-t_{e v}$ diagram can be seen if one plots theoretical tracks on it (cf. dashed curves in Fig. 1b). First of all, the tracks for different masses are well separated for $M_{v}<5$, i.e. during the initial, constant luminosity phase. Thus in this phase different theoretical models can be well tested against observations. However, during the cooling phase the tracks approach one another in the $M_{v}-t_{e v}$ plane. Thus nothing sure can be said about old, lowluminosity objects from this plot.

Another advantage of the discussed diagram is that it uses parameters directly available from observations $\left(M_{v}, R_{P N}\right)$. In other words, the $M_{v}-t_{e v}$ diagram stays closely to observations.

Much of uncertainty when confronting theoretical models to observed PNNi in the $M_{v}-t_{e v}$ plane is due to the fact that the theoretical age, $t_{e v}$ has to be compared to the observed $R_{P N}$ or expansion time, $t_{\exp }=R_{P N} / v_{\exp }\left(v_{\exp }\right.$ - expansion velocity). Observational determinations of $R_{P N}$ can be subject to significant uncertainties, especially for PNe having ill defined outer rims or highly irregular structures, as well as, for compact, badly resolved objects. Errors in $v_{\text {exp }}$ are not negligible either. For many objects there is no measurement and one is forced to use a sort of typical $v_{\text {exp }}$, usually taken as $20 \mathrm{~km} / \mathrm{sec}$.

The theoretical evolutionary time, $t_{e v}$, depends on three factors: nuclear burning rate, zero-age point (moment when the $\mathrm{PN}$ formation is finished) and mass loss rate. The first process is rather well known from the stellar evolution theory. Unfortunately, the other two factors are taken from simplified and somewhat arbitrary considerations which results in significant uncertainties in the theoretical tracks. This problem is discussed in a poster of Górny (see this volume). It appears that within a reasonable range of parameters defining zero-age point and PNN wind rate the uncertainty in $\log t_{e v}$ is typically within 0.3 .

Similarly as in the H-R diagram, the PN distances introduce substantial uncertainties in the $M_{v}-t_{e v}$ plane. It is, however, interesting to note that systematic errors in distances affect the observed positions in the $M_{v}-t_{e v}$ plot in an opposite sense than in the H-R diagram. For instance, increasing distances shifts the observed points in the $M_{v}-t_{e v}$ diagram towards tracks of lower masses whereas in the H-R diagram the result is to get higher $L_{\star}$ and, usually, higher PNN masses. This effect can be used as a test of different distance scales (Tylenda \& Stasińska 1989).

\section{Effects of the Zanstra method and the Shklovsky distances}

The Zanstra method is the simplest and most widely used method for estimating $T_{\star}$, similarly as the Shklovsky method for the PN distances. It is therefore instructive to investigate possible uncertainties and systematic effects introduced by these two methods while constructing observational diagrams.

For this purpose let us consider a simple scenario in which a model PNN is surrounded by a $0.2 M_{\odot}$ nebula expanding with $20 \mathrm{~km} / \mathrm{sec}$ and having a form of a spherically symmetrical shell of a constant thickness $\Delta R / R=0.3$. At a given time moment the $\mathrm{H}$ ionization structure of the nebula can be calculated from simple (Strömgren) considerations adopting that the PNN radiates as a blackbody. This 
allows to estimate the $H \beta$ flux and, subsequently, the HI Zanstra temperature and luminosity. Form the $H \beta$ flux and the extension of the $H^{+}$zone in the model PN one can simulate observational determination of the Shklovsky distance and its effect on various parameters, such as $L_{\star}, M_{v}, t_{e v}$.
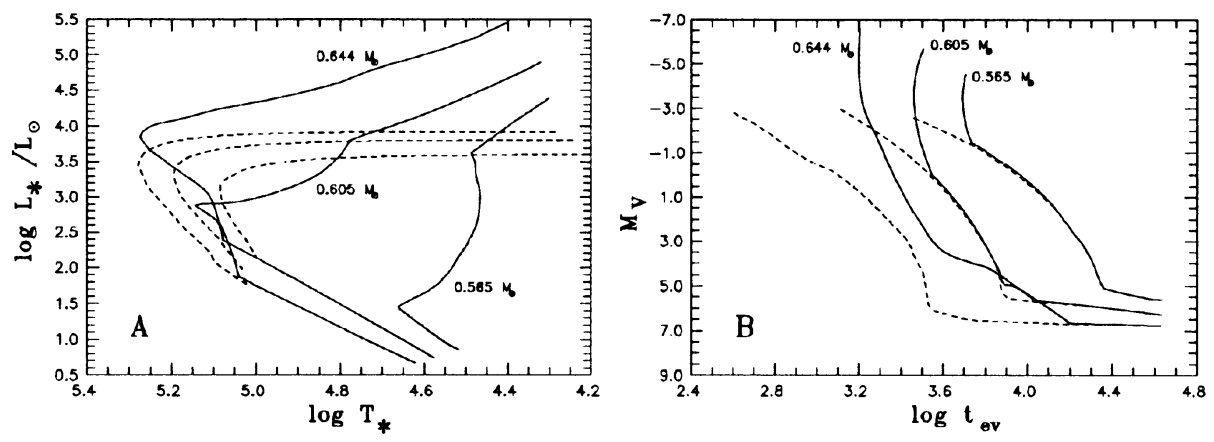

Fig. 1. Effects of the HI Zanstra method and the Shklovsky distances on the observed evolution of $P N N i$ in the H-R diagram (a) and in the $M_{v}-t_{e v}$ diagram (b). Dashed curves - original Schönberner's tracks. Full curves - observed evolution. Curves are labeled with PNN masses.

The results are displayed in Fig. 1. Dashed curves show the original Schönberner's tracks (Schönberner 1979, 1983; Blöcker \& Schönberner 1990). Full curves present the "observed" evolution from our simulations.

The curves for lower mass PNNi (particularly $0.565 M_{\odot}$ ) in Fig. 1a illustrate quantitatively what has been said in Sect. 1 about the danger of using the observed $H \beta$ (or radio) flux as an indicator of $L_{\star}$. A low mass, slowly evolving PNN leaves enough time for its PN to expand considerably. When the PNN starts emitting ionizing photons the diffuse PN quickly becomes totally ionized and the $H \beta$ line measures only a fraction of the ionizing flux. Consequently, the PNN appears as a very low $T_{\star}$ and very low $L_{\star}$ object, i.e. at a position where no PNN is expected according to the stellar evolution theory.

Systematic errors due to the Shklovsky method are stronger for more massive PNNi (see particularly $0.644 M_{\odot}$ in Fig. 1), especially in initial phases. These PNNi evolve fast and they start ionizing the nebular gas very early when their PNe are compact and dense. Consequently, $M_{\text {ion }}$ is much smaller than $0.2 M_{\odot}$ and the Shklovsky method considerably overestimates the distance. As a result the tracks of more massive PNNi are moved upwards in the H-R diagram (Fig. 1a) and rightupwards in the $M_{v}-t_{e v}$ plot (Fig. 1b).

An interesting observation can be made upon comparing Figs. 1a and 1b. As a result of the Zanstra method and the Shklovsky distances the "observed" positions of $\mathrm{PNNi}$ in the $\mathrm{H}-\mathrm{R}$ diagram are much more dispersed than the purely theoretical tracks (cf. full and dashed curves in Fig. 1a). Consequently, the "observed" PNN mass distribution derived from Fig. 1a would be wider than the real one. The effect in the $M_{v}-t_{e v}$ plane is, however, qualitatively different. Due to the Shklovsky distances more massive PNNi move towards low mass tracks. Thus in this case the "observed" PNN mass distribution would show less high mass objects than in 
reality. Perhaps this is the main reason why the PNN mass distributions derived from the $M_{v}-t_{e v}$ diagram (e.g. Schönberner 1981; Weidemann 1989) are usually narrower and peaked at lower masses than those obtained from the H-R diagram (e.g. Kaler 1983; Kaler et al. 1990).

As can be seen from Fig. 1, observational methods can substantially modify PNN positions in the diagrams. It is not easy to correct for these effects in a real situation. Hence an idea, so far explored by Stasińska \& Tylenda (1990) and Tylenda et al. (1991), to apply exactly the same methods of deriving parameters for both, observed objects and theoretical evolving PNNi surrounded by expanding model $\mathrm{PNe}$. In this way the theoretical tracks are brought much closer to the observations and systematic errors due to observational methods are minimized. Unfortunately this procedure introduces uncertainties due to the model PNe.

\section{Distance-independent diagrams}

As mentioned above, investigations of PNNi very often suffer from uncertainties in the distances. Hence importance of analyses using observational parameters independent of the distances.

Tylenda \& Stasińska (1989) have proposed a distance-independent diagram which plots $T_{\star}$ against a parameter $f=L_{v} /\left(4 \pi t_{e v}{ }^{2}\right)$ where $L_{v}$ is the PN$N$ luminosity in the $V$ band. For observed objects, adopting that $t_{e v}=t_{\text {exp }}$ one gets $f=\left(F_{v} v_{\text {exp }}{ }^{2}\right) / \theta^{2}$ where $F_{v}$ denotes the PNN flux observed (corrected for extinction) in the $V$ band and $\theta$ is the observed angular radius of the PN.

The $T_{\star}-f$ plane combines observational parameters used in the $\mathrm{H}-\mathrm{R}$ and $M_{v}$ - $t_{e v}$ diagrams. Hence many remarks from Sect. 1 and 2 are valid here, as well. Similarly as in the H-R diagram, uncertainties in $T_{\star}$ affect the observed positions in the $T_{\star}-f$ plot. Problems of the $M_{v}-t_{e v}$ diagram (zero-age point, mass loss, observational measuring of $\theta$ and $v_{\text {exp }}$ ) introduce uncertainties while comparing the $f$ values for observed objects and theoretical tracks. The $T_{\star}-f$ diagram, similarly as $M_{v}-t_{e v}$, is particularly suitable for investigating PNNi with active shell sources. During the cooling phase the tracks of different masses follow similar paths and an analysis becomes very uncertain.

Several distance-independent diagrams have recently been investigated by Zhang $\&$ Kwok (1992). Most useful for testing of the PNN evolution is a plot of $T_{b}$ versus $T_{\star}$, where $T_{b}$ is the $\mathrm{PN}$ radio brightness temperature. This diagram greatly reduces problems due to extinction. There are, however, several sources of uncertainties while analyzing this diagram. Firstly, as mentioned in Sect. 1, determination of $T_{\star}$ is far from being a simple problem. Secondly, uncertainties and errors while measuring PN radii (ill defined PN rims, irregular objects, small, badly resolved PNe) can seriously affect the derived brightness temperature as $T_{b} \sim \theta^{-2}$. Thirdly, when calculating $T_{b}$ for a theoretical track one has to adopt a PN model. Bearing in mind numerous problems while modelling $\mathrm{PN}$ formation and evolution one realizes that the positions of theoretical tracks in the $T_{b}-T_{\star}$ plane are uncertain.

The PNN evolution can also be studied using other diagrams which I cannot discuss because of lack of space. Let me mention that Schönberner (1986) has attempted to constrain PNN models from a plot of the nebular excitation versus $M_{v}$. 
Szczerba has applied a $H e I I \lambda 4686$ line intensity histogram (Szczerba 1987) and a plot of the nebular ratio $H e I I \lambda 4686 / H \beta$ versus $F(H \beta) / F_{P N N}(\lambda 4861)$ where $F_{P N N}(\lambda 4861)$ is the PNN continuum flux at $4861 \AA$ (Szczerba 1990). A PN luminosity function in $H \beta$ and [OIII] can also be used as shown by Jacoby (1989) and Stasińska et al. (1991b). Finally, studies of PN line intensities can provide constraints to PNN models as well, as in Vilkoviskii et al. (1983) and Stasińska (1989).

\section{Observed PNN evolution and masses}

This section summarizes the results obtained by different authors concerning the evolution and masses of the Galactic PNNi. Results for other galactic systems are discussed elsewhere (e.g. Dopita in this volume).

At the very beginning it should be mentioned that it is rather difficult and somewhat dangerous to compare and draw conclusions from results obtained in different papers. The reason is that different authors use different diagrams, different distance scales, different PN samples in which observational selection effects can also be different. My feeling is that much of difference between individual determinations is indeed due to these factors.

Schönberner (1981) has investigated the problem of PNN masses using the $M_{v}$ - $t_{e v}$ diagram and the Shklovsky distances. He has concluded that most of PNNi burn hydrogen quiescently and found a very narrow distribution with an average PNN mass of $0.58 M_{\odot}$ and $85 \%$ of the objects within $\pm 0.03 M_{\odot}$.

Heap \& Augensen (1987) have used the same type of diagram but with $U V$ PNN magnitudes and Daub's (1982) distances. They have obtained a PNN mass distribution much wider than Schönberner with a mean value of $\sim 0.60 M_{\odot}$ and an important high mass tail extending up to $\sim 0.8 M_{\odot}$.

Weidemann (1989) has argued that the Daub's distance scale is too small. Using the same observational material as Heap \& Augensen but with modified distances he has derived a distribution essentially the same as Schönberner.

Kaler (1983) has explored the H-R diagram for investigating the PNN masses. His study has been limited to large PNe for which the Shklovsky method is expected to give a correct distance scale. The Kaler's distribution is wider than the Schönberner's with $\sim 40 \%$ of objects above $0.6 M_{\odot}$.

The Kaler's study has been extended in Kaler et al. (1990) using new observational data. They have obtained a still wider distribution with $\sim 40 \%$ above $0.7 M_{\odot}$.

The H-R diagram has also been investigated by Pottasch (1984) and Gathier \& Pottasch (1989) who adopted the so-called individual distances. They have obtained a large number of cool PNNi at low luminosities $\left(<10^{3} L_{\odot}\right)$ which would imply masses of $\sim 0.5 M_{\odot}$. This is in conflict with the theoretical models which predict that $\mathrm{PNe}$ with central stars $<0.55 M_{\odot}$ should not be observed because of too a long time scale of their post-AGB evolution (Schönberner 1983). Gathier \& Pottasch conclude that low-mass PNNi evolve much faster than theoretically predicted.

Tylenda \& Stasinska (1989) have analyzed the PNN evolution in three diagrams, i.e. H-R, $M_{v}-t_{e v}$ and $T_{\star}-f$. They have found that the individual distances lead to inconsistent results suggesting that these distances are often underestimated. 
Instead, the Shklovsky distances have given consistent results from different diagrams. The authors conclude that most of PNNi have masses between $0.55 M_{\odot}$ and $0.65 M_{\odot}$ and that the $\mathrm{H}$-burning $\mathrm{PNN}$ models better reproduce the observations than the He-burners.

Stasińska \& Tylenda (1990) have used the same diagrams as in Tylenda \& Stasińska (1989) but with theoretical tracks modified by the presence of a PN and observational effects due to the Zanstra and Shklovsky methods. They have obtained the PNN masses between $0.55 M_{\odot}$ and $\sim 0.7 M_{\odot}$ and a peak mass at $0.61 M_{\odot}$.

Zhang \& Kwok (1992) have derived a strongly asymmetric PNN mass distribution from their distance-independent $T_{b}-T_{\star}$ diagram. The distribution peaks at $0.60 M_{\odot}$ and has a high mass tail extending up to $0.85 M_{\odot}$.

From a $H e I I \lambda 4686$ line intensity histogram and a plot of $H e I I \lambda 4686 / H \beta$ versus $F(H \beta) / F_{P N N}(\lambda 4861)$ Szczerba $(1987,1990)$ has concluded that the PNN mass distribution is strongly peaked at $0.60 M_{\odot}$ and that the PNNi mostly burn hydrogen.

\subsection{Galactic Bulge}

Investigations of $\mathrm{PNe}$ in the Galactic bulge are important because of two reasons. Firstly, this is the only sample of PNe in the Galaxy for which we have fairly well known distances. Secondly, it is expected that these objects might be somewhat different from the rest of the PNe in the Galaxy.

Webster (1988) compared the excitation classes of PN samples in the Galactic bulge and in the Magellanic Clouds. She has concluded that the bulge has more low-mass progenitor stars than the LMC and the solar neighbourhood.

Zijlstra \& Pottasch (1989) and Pottasch \& Acker (1989) have constructed H-R diagrams for the Galactic bulge PNNi. In both studies the PNN luminosities have been calculated from the $H \beta$ (or radio) fluxes as the authors argue that the $\mathrm{PNe}$ are opaque. The resulting diagrams show most of the objects to be of low $L_{\star}$ and low $T_{\star}$ which cannot be explained by the existing PNN models. Pottasch \& Acker conclude that high mass PNNi evolve much slower and low mass PNNi much faster than predicted from the theory.

Tylenda et al. (1991) have analyzed several diagrams with theoretical tracks modified for observational effects due to the presence of a nebula. They have obtained a mean PNN mass of $0.593 M_{\odot}$ with a standard deviation of $0.025 M_{\odot}$. This can be compared to the value of $0.615 \pm 0.036 M_{\odot}$ found for a sample of Galactic disc PNe by Stasiniska \& Tylenda (1990) using a similar procedure of analysis. This would suggest that the bulge PNNi are less massive. However, the conclusion is not sure as the selection effects occurring in the two samples are not the same.

From an analysis of [OIII] and $H \beta$ luminosity functions for the bulge PNe Stasińska et al. (1991b) have obtained a mean PNN mass of $0.57-0.60 M_{\odot}$ and a standard deviation of $0.04-0.05 M_{\odot}$.

Mendez (1992) has estimated the PNN luminosities in the bulge using observed PNN magnitudes and bolometric corrections derived from the Stoy method. He has obtained a mean $\log L_{\star} / L_{\odot}=3.86$ which corresponds to $0.625 M_{\odot}$ as a mean PNN mass (according to the Schönberner's models). Comparison of the results of 
Mendez with those of Tylenda et al. (1991) shows the effect of different methods for deriving extinction (Balmer decrement in the former, radio/ $H \beta$ in the latter).

\subsection{PNN MASSES FROM SPECTROSCOPIC STUDIES}

The most sophisticated method for determining PNN masses is the one elaborated by Mendez et al. (1988), based on a model atmosphere analysis of observed PNN absorption line profiles (see also Kudritzki in this volume). The method has a great advantage of being independent of distances. However, it can be applied only to bright central stars showing little nebular contamination for which accurate line profiles can be obtained spectroscopically.

Mendez et al. $(1988,1990)$ have analyzed 23 PNNi using non-LTE plane-parallel model atmospheres. They have obtained PNN masses substantially higher from those found in other studies summarized above. A mean value of their masses is $0.70 M_{\odot}$ with a standard deviation of $0.09 M_{\odot}$.

Recently, Mendez et al. (1992) have reanalyzed their observational material using more realistic non-LTE model atmospheres including spherical extension and stellar winds. As a result the PNN gravities have been somewhat reduced leading to slightly lower - but still quite high - masses, i.e. $0.65 \pm 0.05 M_{\odot}$.

It is often argued that the reason why Mendez et al. find high PNN masses is that they picked up the most luminous and thus the most massive PNNi. However, this argument does not seem to be convincing. The fact that a sample contains objects apparently brightest does not necessarily imply that they are intrinsically most luminous. In fact it can be argued on theoretical grounds that within luminous $\mathrm{PNNi}$ it is more probable to find a low mass object than a high mass one.

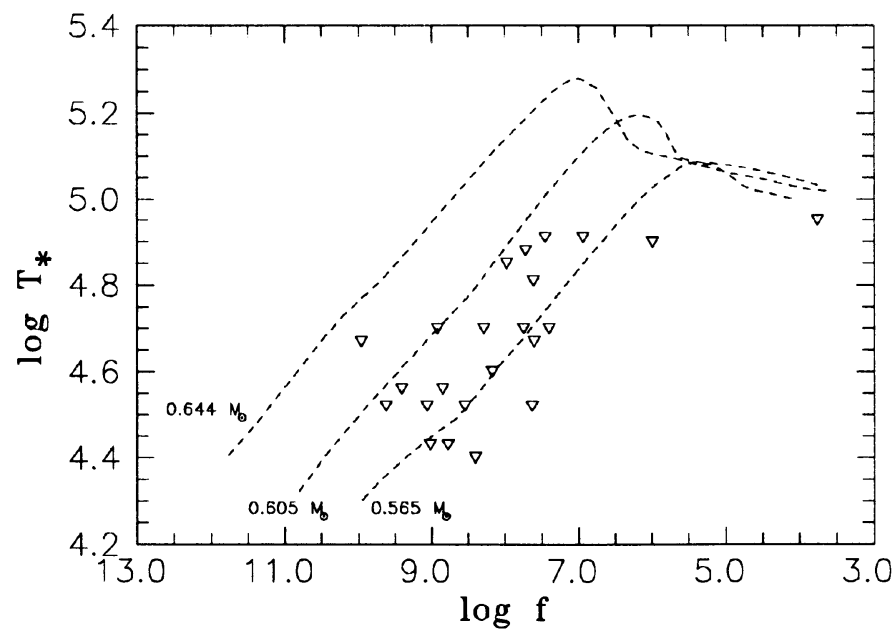

Fig. 2. $T_{\star}$ - $f$ diagram for the PNN sample of Mendez et al. (1992). Dashed curves - theoretical tracks of Schönberner labeled with PNN masses. Triangles - observed objects.

Fig. 2 shows the sample of Mendez et al. in the $T_{\star}-f$ diagram. The values 
of $T_{\star}$ have been taken from Mendez et al. (1992). Observational data necessary to calculate $f$ have been taken from Acker et al. (1992).

As can be seen from Fig. 2, there is nothing exceptional in the positions of PNNi from the sample of Mendez et al. They are fairly typical for other PNNi (see e.g. Fig. 3 in Tylenda \& Stasińska 1989). A similar conclusion, although from a different diagram, has been drawn by Szczerba (1990). An estimation of the PNN masses from Fig. 2 gives $0.58 \pm 0.02 M_{\odot}$.

The consequences of the above result are the following. If the PNN masses derived by Mendez et al. are correct than all the PNNi are more massive and more luminous than derived in other works. In addition, as concluded by McCarthy et al. (1990), the theoretical evolutionary time scales are wrong. The PNNi, after having formed their nebulae, evolve much slower than predicted. If, however, as found in other studies, the present PNN models are roughly correct than there is something wrong or missing in the present model atmosphere analyses which leads to a systematic overestimate of the PNN masses.

Acknowledgement. This work was supported in part from grant no. 2-2114-92-03 financed by Polish Committee for Scientific Research.

\section{References}

Abell (i.O., 1966, ApJ 144, 259

Acker A., Ochsenbein F., Stenholm B., Tylenda R., Marcout J., Schohn C., 1992, Strasbourg-ESO Catalogue of Galactic Planetary Nebulae (ESO publ.)

Blöcker T., Schönberner D., 1990, A\&A 240, L11

Cahn J.H., Kaler J.B., Stanghellini L., 1992, A\&AS 94, 399

Daub C.T., 1982, ApJ 260, 612

Gathier R., Pottasch S.R., 1989, A\&A 209, 369

Heap S.R., Augensen H.J., 1987, ApJ 313, 268

Jacoby G.H., 1989, ApJ 339, 39

Kaler J.B., 1983, ApJ 271, 188

Kaler J.B., Shaw R.A., Kwitter K.B., 1990, ApJ 359,392

McCarthy J.K., Mould J.R., Mendez R.H., Kudritzki R.P., Husfeld D., Herrero A., Groth H.G., 1990, ApJ 351, 230

Mendez R.H., 1992, A\&A in press

Mendez R.H., Kudritzki R.P, Herrero A., Husfeld D., Groth, H.G., 1988, A\&A 190, 113

Mendez R.H., Herrero A., Manchado A., 1990, A\&A 229, 152

Mendez R.H., Kudritzki R.P., Herrero A., 1992, A\&A in press

Pottasch S.R., 1984, Planetary Nebulae, Reidel, Dordrecht

Pottasch S.R., Acker A., 1989, A\&A 221, 123

Schönberner D., 1979, A\&A 79, 108

Schönberner D., 1981, A\&A 103, 119

Schönberner D., 1983, ApJ 272, 708

Schönberner D., 1986, A\&A 169, 189

Schönberner D., Tylenda R., 1990, A\&A 234, 439

Stasińska G., 1989, A\&A 213, 274

Stasińska G., Tylenda R., 1990, A\&A 240, 467 
Stasińska G., Tylenda R., Acker A., Stenholm B., 1991a, A\&A 247, 173

Stasińska G., Fresneau A., da Silva Gameiro G.F., Acker A., 1991b, A\&A 252, 762

Stasińska G., Tylenda R., Acker A., Stenholm B., 1992, A\&A in press

Szczerba, R., 1987, A\&A 181, 365

Szczerba, R., 1990, A\&A 237, 495

Tylenda R., Stasińska G., 1989, A\&A 217, 209

Tylenda R., Stasińska G., Acker A., Stenholm B., 1991, A\&A 246, 221

Tylenda R., Acker A., Stenholm B., Köppen J., 1992, A\&AS in press

Vilkoviskii E.V., Kondrateva L.N., Tambotseva L.V., 1983, Sov. Astron. 27, 194

Webster B.L., 1988, MNRAS 230,377

Weidemann, V., 1989, A\&A 213, 155

Wood P.R., Faulkner D.J., 1986, ApJ 307, 659

Zhang C.Y., Kwok S., 1992, A\&A in press

Zijlstra A.A., 1990, A\&A 234, 387

Zijlstra A.A., Pottasch S.R., 1989, A\&A 216, 245 